\title{
Observation of Magnetic Anisotropy in Bulk GaMnN:Mg Crystals
}

\author{
J. $\operatorname{GosK}^{a, b, *}, \mathrm{M} \cdot \mathrm{ZAJAZC}^{a}, \mathrm{M} \cdot \mathrm{KAmińsKA}^{a}, \mathrm{~A} \cdot \operatorname{TwARDOWSKI}^{a}$, \\ I. Grzegory ${ }^{c}$, M. BoćKowski ${ }^{c}$ And S. Porowski ${ }^{c}$ \\ ${ }^{a}$ Institute of Experimental Physics, Warsaw University \\ Hoża 69, 00-681 Warsaw, Poland \\ ${ }^{b}$ Faculty of Physics, Warsaw University of Technology \\ Koszykowa 75, 00-662 Warsaw, Poland \\ ${ }^{c}$ High Pressure Research Center, Polish Academy of Sciences \\ Sokołowska 29/37, 01-142 Warsaw, Poland
}

\begin{abstract}
Magnetic properties of bulk wurtzite $n$-type GaMnN and highly resistive GaMnN:Mg monocrystals were studied for the magnetic field applied parallel and perpendicular to the crystal hexagonal $c$-axis. Magnetization of both types of samples reveals paramagnetic behavior. However, for $n$-type $\mathrm{GaMnN}$ isotropic magnetization was observed which is in agreement with Mn $d^{5}$ configuration. On the other hand, GaMnN co-doped with $\mathrm{Mg}$ shows large magnetic anisotropy which suggests $\mathrm{Mn}$ to be in nonspherical $d^{4}$ or $d^{3}$ configuration.
\end{abstract}

PACS numbers: 71.55.Eq, 75.30.Gw, 75.50.Pp

\section{Introduction}

Gallium nitride is one of the most promising material for applications in electronic and optoelectronic devices [1]. Moreover, there are theoretical predictions that $\mathrm{GaN}$ doped with a few molar percent of Mn and highly p-type should reveal room temperature ferromagnetism (FM) [2]. This has led to an increased interest in synthesis and magnetic properties of $\mathrm{GaMnN}$ semimagnetic semiconductor. Several methods have been applied to obtain this material so far: ammonothermal [3], molecular beam epitaxy [4], chemical transport [5]. The ferromagnetic (FM) contribution to the magnetization was reported for temperatures ranging up to

*corresponding author; e-mail: gosk@fuw.edu.pl 
$940 \mathrm{~K}$ [4]. However, the available data show that there is no consensus concerning the origin of the observed FM behavior — one should take into account the possibility of formation of FM precipitates $\left(\mathrm{Mn}_{x} \mathrm{~N}_{y}\right.$ or $\left.\mathrm{Ga}_{1-x} \mathrm{Mn}_{x}\right)$ which may not be detectable by X-ray diffraction or electron transmission microscopy measurements, but contribute substantially to the magnetization. Some authors suggest that these compounds exist in their samples $[6,7]$.

In order to understand the magnetic properties of $\mathrm{GaMnN}$ the nature of Mn impurity in GaN should be examined. From the point of view of exchange interactions one of the most important questions is how the carrier concentration influences the charge state of Mn dopant. Previous studies performed on the samples grown by ammonothermal and chemical transport methods indicate that $\mathrm{Mn}$ enters GaN as an ionized acceptor in $d^{5}$ configuration $(S=5 / 2)$ [3]. Since more and more studies show that the $\mathrm{Mn}^{2+/ 3+}$ acceptor level is most likely located in the energy gap of $\mathrm{GaN}[8,9]$, the presence of $\mathrm{Mn}^{2+}$ centers may be the result of $n$-type character of the studied samples. One may then expect that the nature of Mn impurity results from mutual position of $\mathrm{Mn}^{2+/ 3+}$ level and the Fermi level which can be changed by co-doping. In particular, co-doping with $\mathrm{Mg}$, which is an acceptor, should result in lowering of the Fermi level. In order to verify this hypothesis we studied the magnetic properties of bulk $n$-type GaMnN and highly resistive $\mathrm{GaMnN}: \mathrm{Mg}$ samples. In this paper we compare the results of magnetic measurements performed on these two types of samples.

\section{Experimental}

Single GaMnN and GaMnN:Mg crystals were grown by equilibrium high pressure technique from nitrogen solution in liquid gallium. Manganese and magnesium were added into gallium during the growth. The growth was performed under high pressure of $\mathrm{N}_{2}(p \approx 1.5 \mathrm{GPa})$ and temperatures $T \approx 1500^{\circ} \mathrm{C}$. The obtained crystals were platelets with diameter of about few millimeters and thickness of about $100 \mu \mathrm{m}$. GaMnN samples were highly conductive with concentration of free electrons $n \times 10^{20} \mathrm{~cm}^{-3}$ while GaMnN:Mg samples were highly resistive.

Magnetization was measured using a superconducting quantum interference device (SQUID) operating in the temperature range $2-300 \mathrm{~K}$ and magnetic field up to $6 \mathrm{~T}$. The magnetic field was applied in perpendicular or parallel orientation to the GaN hexagonal c-axis. All the GaMnN data were corrected for the diamagnetism of the $\mathrm{GaN}$ host lattice.

\section{Results and discussion}

Representative magnetization data for $n$-type GaMnN plotted as a function of magnetic field and temperature are exemplified in Fig. 1. In general, overall paramagnetic (PM) behavior is observed. At high temperatures magnetization shows 


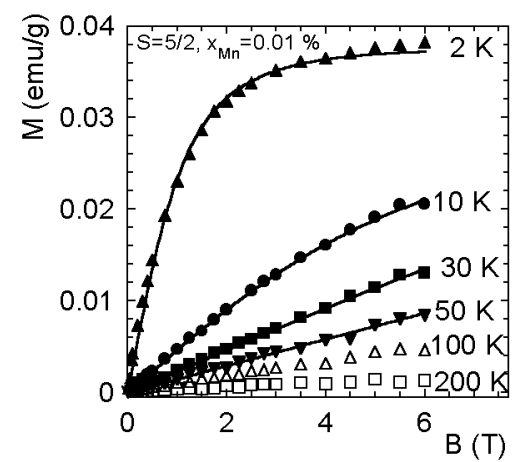

Fig. 1. Representative magnetization of an $n$-type $\mathrm{GaMnN}$ sample plotted as a function of the magnetic field for several temperatures. The data are corrected for diamagnetism of the GaN host lattice. Solid curves represent the fits corresponding to the Brillouin function with $S=5 / 2$.

a linear magnetic field dependence. At low temperatures a pronounced tendency to saturation is observed. A negligible FM contribution (less than 1\%) was also visible in some samples and was attributed to the presence of $\mathrm{Mn}_{x} \mathrm{~N}_{y}$ precipitates. We note that similar behavior was reported for samples grown by ammonothermal and chemical transport methods $[3,5]$. The data can be well described by a Brillouin function with $S=5 / 2$, expected for $d^{5}$ configuration of $\mathrm{Mn}$ ion. The manganese concentration resulting from the fit is below $0.2 \%$. Such concentration is too low to reveal $d-d$ interaction between Mn ions. Thus, we can assume that we are dealing with a system of noninteracting magnetic moments coming from $d^{5}$ configuration. Moreover, as shown in Fig. 2, there is no significant difference between magnetization of the same sample measured at the parallel and perpendicular orientations of magnetic field relatively to the $c$-axis. In other words no magnetic anisotropy was observed for $n$-type GaMnN bulk samples. Such behavior

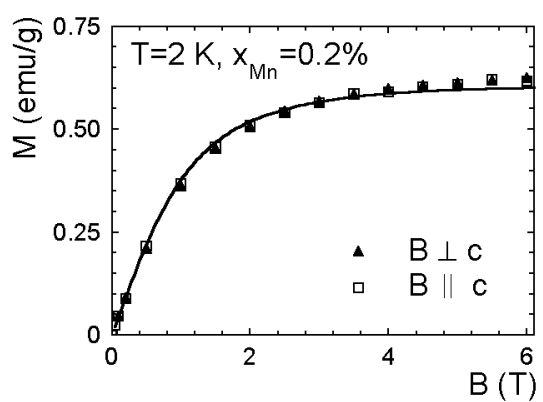

Fig. 2. Results of the magnetization measurements of an $n$-type GaMnN sample performed at parallel and perpendicular orientation of magnetic field to the $c$-axis at $2 \mathrm{~K}$. Solid curve corresponds to the Brillouin function curve with $S=5 / 2$. 
is characteristic of spherically symmetrical $d^{5}$ configuration of Mn ion, which is in agreement with the result obtained from the fit at $2 \mathrm{~K}$.

On the other hand, highly resistive GaMnN:Mg samples show different magnetic properties (Fig. 3). Not only the Brillouin function with $S=5 / 2$ is inadequate to describe the data but a very large magnetic anisotropy appears (Fig. 3). This anisotropy decreases with increasing temperature. At $T=2 \mathrm{~K}$ magnetization measured at magnetic field parallel to the $c$-axis saturates much slower than that for the perpendicular orientation. We recall that similar magnetic anisotropy was also observed for Cr and V-based II-VI hexagonal semimagnetic semiconductors $[10,11]$. This may suggest that $\mathrm{Mn}$ center in GaMnN:Mg is in a nonspherical configuration, presumably $d^{4}$ or $d^{3}$. Such centers suffer strong Jahn-Teller distortion along one of four $\langle 111\rangle$ axes (in the case of $d^{3}$ configuration) or three $\langle 100\rangle$ axes (in the case of $d^{4}$ configuration). The observed anisotropy most probably originates from different distributions of non-equivalent Jahn-Teller centers in two orientations of magnetic field. The anisotropy of effective mass hole bound to the Mn acceptor $\left(\mathrm{Mn}^{2+}+\mathrm{h}\right)$ may also be a possible reason of the observed magnetic behavior. However, in the first approach we neglect the influence of carriers. This problem will be analyzed within the crystal field model in more detail elsewhere.

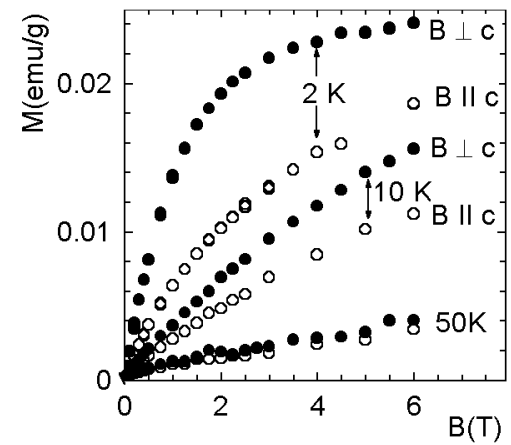

Fig. 3. Magnetization of GaMnN:Mg measured as a function of magnetic field at different temperatures. Magnetic field is applied in perpendicular (closed circles) and parallel (open circles) orientations relative to the $c$-axis. A strong magnetic anisotropy is visible.

The observed magnetic properties could be understood assuming that $\mathrm{Mn}$ enters GaN as substitutional $\mathrm{Mn}^{2+}$ ( $d^{5}$ configuration), leading to a Brillouin-type paramagnetism. On the other hand, acceptor-type co-dopants (e.g. Mg) reduce the number of $d$-shell electrons, yielding $d^{4}$ or $d^{3}$ configuration. Consequently, magnetic anisotropy appears. This suggestion was confirmed by EPR experiment which indicated that $\mathrm{Mn}^{2+}\left(d^{5}\right)$ configuration is not the majority one in GaMnN:Mg sample [12]. 


\section{Conclusions}

Magnetic properties of bulk $n$-type GaMnN and highly resistive GaMnN:Mg sample were studied for the magnetic field applied parallel and perpendicular to the hexagonal $c$-axis. Contrary to the $n$-type samples a strong magnetic anisotropy was observed for GaMnN:Mg sample, which suggests that Mn enters GaN:Mg as a nonspherical center in $d^{3}$ or $d^{4}$ configuration.

\section{Acknowledgments}

This work was partially supported by the State Committee for Scientific Research under grant 115/E-343/SPUB-M/5.PR UE/DZ 255/2001-2003 and partially under the project FENIKS (G5RD-CT-2001-2001-00535).

\section{References}

[1] S.C. Jain, M. Willander, J. Narayan, R. van Overstraeten, J. Appl. Phys. 87, 965 (2000); O. Ambacher, J. Phys. D, Appl. Phys. 31, 2653 (1998); S. Nakamura, in: GaN and Related Materials, Ed. S.J. Pearton, Gordon and Breach, New York 1997, p. 471; S. Nakamura, in: Semiconductors and Semimetals, Vol. 50, GaN, Eds. J. Pankove, T.D. Moustakas, Academic, New York 1998, p. 431.

[2] T. Dietl, H. Ohno, F. Matsukura, J. Cibert, D. Ferrand, Science 287, 1019 (2000).

[3] M. Zając, R. Doradziński, J. Gosk, J. Szczytko, M. Lefeld-Sosnowska, M. Kamińska, A. Twardowski, M. Palczewska, E. Grzanka, W. Gębicki, Appl. Phys. Lett. 78, 1276 (2001)

[4] S. Sonoda, S. Shimizu, T. Sasaki, Y. Yamamoto, H. Hori, J. Cryst. Growth 237-239, 1358 (2002).

[5] T. Szyszko, G. Kamler, B. Strojek, G. Weisbrod, S. Podsiadło, L. Adamowicz, W. Gębicki, J. Szczytko, A. Twardowski, K. Sikorski, J. Cryst. Growth 233, 631 (2001).

[6] M. Zając, J. Gosk, E. Grzanka, M. Kamińska, A. Twardowski, T. Szyszko, B. Strojek, S. Podsiadło, J. Appl. Phys. 93, 4715 (2003).

[7] S. Dhar, O. Brandt, A. Trampert, L. Däweritz, K.J. Friedland, K.H. Ploog, J. Keller, B. Beschoten, G. Güntherodt, Appl. Phys. Lett. 82, 2077 (2003).

[8] R.Y. Korotkov, J.M. Gregie, B.W. Wessels, Appl. Phys. Lett. 80, 1731 (2002).

[9] T. Graf, M. Gjukic, M.S. Brandt, M. Stutzmann, O. Ambacher, Appl. Phys. Lett. 81, 5159 (2002).

[10] M. Herbich, W. Mac, A. Twardowski, K. Ando, D. Scalbert, A. Petrou, M. Demianiuk, Phys. Rev. B 58, 1912 (1998).

[11] M. Herbich, W. Mac, A. Twardowski, M. Demianiuk, Phys. Rev. B 59, 2726 (1999).

[12] A. Wołoś, M. Palczewska, Z. Wilamowski, M. Kamińska, A. Twardowski, M. Boćkowski, I. Grzegory, S. Porowski, Acta Phys. Pol. A 104, (2003). 